\title{
Correction to: Somato-Dendritic Secretion of Neuropeptides
}

Colin H. Brown, Mike Ludwig, and Javier E. Stern

\section{Correction to:}

Chapter 4 in: J. R. Lemos, G. Dayanithi (eds.), Neurosecretion: Secretory Mechanisms, Masterclass in Neuroendocrinology 8, https://doi.org/10.1007/978-3-030-22989-4_4

The original version of Chapter 4 was inadvertently published with only the second affiliation of the author Mike Ludwig, but his first affiliation was missing. This has now been corrected. 